\title{
Incorporando dispositivos de radio definida por software en la materia de Comunicaciones Digitales: del grupo piloto a la gran clase*
}

\author{
Carmen Botella ${ }^{1}$, Antonio Soriano-Asensi ${ }^{1}$, Jaume Segura-Garcia ${ }^{1}$, Joaquin Perez ${ }^{2}$, \\ Santiago Felici-Castell ${ }^{1}$, Enrique Navarro-Camba ${ }^{1}$, Miguel García-Pineda ${ }^{1}$ y Mario \\ Montagud $^{1}$ \\ ${ }^{1}$ Departament d'Informàtica, Universitat de València. Av. de la Universitat s/n, 46100 Burjassot \\ (SPAIN) \\ ${ }^{2}$ Departament d'Enginyeria Electrònica, Universitat de València. Av. de la Universitat s/n, 46100 \\ Burjassot (SPAIN)
}

\begin{abstract}
Educational innovation is a process that extends beyond an academic year. In its development, the stages of planning, implementation, evaluation and feedback are distinguished in order to identify aspects that could be improved. In this contribution, we present the work developed over the last two years in the subject Communication Theory (Digital Communications), Degree in Telematic Engineering, from the University of Valencia. Specifically, software defined radio devices have been incorporated in the laboratories as a tool to bring students closer to more realistic practices. The paper describes the adaptation process of a laboratory session, which was initially developed by a small pilot group in the academic year 2018-2019, for an experimental laboratory group in the academic year 2019-2020. In order to evaluate the impact of the innovation, the involvement of the students is quantified, comparing the results of the experimental group with two control groups that have continued with the standard simulated sessions. The results indicate that the impact has been positive on the students' ability to face new challenges and on their perception of the relevance of the activities they carry out, although this improvement is not reflected in their ability to focus on them.
\end{abstract}

Keywords: software defined radio, SDR, Digital Communications, active learning.

\section{Resumen}

La innovación educativa es un proceso que se extiende más allá de un curso académico. En su desarrollo se distinguen las etapas de planificación, implementación, evaluación

\footnotetext{
*Este trabajo ha sido financiado por el "Vicerectorat d'Ocupació i Programes Formatius" de la Universitat de València a través del proyecto UV-SFPIE_PID19-1097673
} 
y realimentación, para identificar aspectos susceptibles de mejora. En esta contribución presentamos el trabajo desarrollado en los dos últimos cursos en la asignatura Teoría de la Comunicación (materia de Comunicaciones Digitales), Grado en Ingeniería Telemática, de la Universitat de València. Concretamente, se incorporan dispositivos de radio definida por software en los laboratorios como herramienta para conseguir unas prácticas más realistas. El artículo describe el proceso de adaptación de una sesión de laboratorio, que inicialmente fue desarrollada por un grupo piloto reducido en el curso 2018-2019, para un grupo experimental de laboratorio en el curso 2019-2020. Para evaluar el impacto de la innovación se cuantifica la implicación del alumnado, comparando los resultados del grupo experimental con respecto a dos grupos de control que han continuado con las sesiones simuladas estándar. Los resultados indican que el impacto ha sido positivo en la capacidad de los estudiantes para afrontar nuevos retos y en su percepción de la relevancia de las actividades que realizan, aunque esta mejora no se refleje en su capacidad de centrarse en ellas.

Keywords: radio definida por software, SDR, Comunicaciones Digitales, aprendizaje basado en proyectos.

\section{Introducción}

La radio definida por software (SDR, a partir de sus siglas en inglés, Software Defined Radio) (Mitola 1992) se basa en el uso de dispositivos que digitalizan la señal de radiofrecuencia y envían las muestras a un ordenador, donde se procesan para extraer información ${ }^{1}$. Estos dispositivos han supuesto una revolución en la enseñanza de telecomunicaciones, al permitir implementar muchos componentes de los sistemas mediante programas informáticos, que además pueden reconfigurarse en tiempo real (Bilén y Col. 2014; Stewart 2015; Gil Jiménez 2017). Aunque el concepto de SDR surgió como una evolución natural de la necesidad de digitalizar las redes de telecomunicaciones, en la actualidad permite la implementación de estándares como DVB-T, WiFi (IEEE 802.11), Bluetooth o LTE (4G), cuyos bloques funcionales muestran una buena superposición con los contenidos de las asignaturas impartidas en Grados relacionados con las Telecomunicaciones. La SDR se ha convertido, por tanto, en un recurso educativo fundamental (Wyglinski 2016; Rice y Col. 2019).

En esta contribución se detalla el trabajo de innovación docente realizado en los dos últimos cursos académicos en la asignatura Teoría de la Comunicación (TC), ubicada en el primer cuatrimestre de tercer curso del Grado en Ingeniería Telemática de la Universitat de València (GIT-UV). Esta asignatura (6 ECTS), perteneciente a la materia de Comunicaciones Digitales (24 ECTS), se centra en los distintos bloques que conforman los sistemas de comunicaciones digitales, es decir, el transmisor (codificación de fuente, codificación de canal, modulación), el canal (capacidad del canal y límites fundamentales) y el receptor (cálculo de probabilidades de error). Este trabajo se enmarca en la línea de introducir la tecnología SDR en los laboratorios del GIT-UV, con el objetivo de acercar la docencia hacia una experimentación más realista de los sistemas de comunicaciones, cumpliendo los requisitos especificados por acreditaciones como EUR-ACE ${ }^{2}$ (donde se exigen proyectos transversales a distintas asignaturas y se intenta cuantificar la dimensión de "lo que se espera que los estudiantes sean capaces de hacer"). Concretamente, en el curso 2015-2016 se empezó a trabajar

\footnotetext{
${ }^{1}$ https://www.itu.int/pub/R-REP-SM. 2152-2009

2 https://www .enaee.eu/eur-ace-system/
} 
con la plataforma Universal Software Radio Peripheral (USRP) (Segura 2016). Sin embargo, estos dispositivos tienen un alto coste económico (alrededor de 1000 euros por dispositivo) y no es viable montar sesiones de laboratorio en asignaturas de grado con hasta 60 estudiantes. Posteriormente, en el curso 2018-2019, el resultado principal de la innovación fue la incorporación de soluciones de bajo coste como el RTL-SDR ${ }^{3}$ (alrededor de 33 euros por dispositivo), y de coste medio como el HackRF $\mathrm{One}^{4}$ (300 euros por dispositivo), junto con el uso de una herramienta de software de código abierto (GNURadio) para gestionarlos, de manera que ya se disponía de bancos de trabajo con un coste asumible para asignaturas con un número elevado de matriculados. Además de la implementación de estos bancos de trabajo, se exploró la metodología de Aprendizaje Basado en Proyectos (ABP), de forma que los estudiantes del Máster en Ingeniería de Telecomunicación de la Universitat de València (MITUV), desarrollaban proyectos que posteriormente se incorporaban como sesiones de laboratorio en el GIT-UV (Soriano-Asensi 2019).

En el caso de la asignatura TC, en el curso académico 2018-2019 se planteó un proyecto piloto en el que un número reducido de estudiantes desarrollaba una sesión de laboratorio basada en SDR (mediante el entorno GNURadio) a partir de unas indicaciones básicas propuestas por el profesorado. En el curso académico 2019-2020, esta sesión se ha ampliado a la totalidad de un grupo de laboratorio. Cabe destacar que en los proyectos de innovación anteriormente mencionados, la evaluación de la innovación se realizaba mediante encuestas a los estudiantes participantes (siempre voluntarios, y por tanto, con un sesgo a priori positivo hacia la acción de innovación), o bien cuantificando posibles mejoras en las notas de laboratorio o en las encuestas de satisfacción del alumnado realizadas por el sistema de control de calidad de la titulación. Sin embargo, este tipo de evaluación no es sistemática, y en trabajos como (Soriano-Asensi 2019) se ha comprobado que hay factores externos que influyen en los resultados obtenidos, como, por ejemplo, el número de estudiantes registrados en los grupos de laboratorio, de manera que el docente obtiene resultados peores en los grupos con más estudiantes.Por estos motivos, en esta contribución se persigue la mejora del mecanismo de evaluación, que se centra en el análisis de la actitud del estudiantado en el aula, para lo cual se empleará una encuesta basada en (Schaufeli y Bakker 2004; GarcíaRos y Col. 2018), que analiza el grado de implicación de los estudiantes a partir de tres factores: energía, absorción y dedicación (definidos en la sección 4). Para sistematizar la evaluación, la práctica basada en SDR se imparte en un grupo experimental, mientras que el resto de los grupos de laboratorio se consideran de control y continúan con las prácticas simuladas estándar. Ambas agrupaciones constan de un número similar de componentes.

El resto del artículo se organiza como sigue: en la sección 2 se detallan los objetivos planteados en la actividad de innovación docente. El desarrollo de la innovación se explica en la sección 3. Los resultados obtenidos con la experiencia y su traslación a los laboratorios en GIT-UV se discuten en la sección 4. La sección 5 resume lo aprendido en esta experiencia.

\footnotetext{
${ }^{3}$ https://www.rtl-sdr.com

${ }^{4}$ https://greatscottgadgets.com/hackrf
} 


\section{Objetivos}

Los proyectos planteados con dispositivos SDR pueden integrar de forma transversal conceptos incluidos en materias como electromagnetismo, radiofrecuencia, comunicaciones, procesado digital de la señal, sistemas embebidos, programación e ingeniería de sistemas, etc. La enseñanza de estas materias se suele impartir tradicionalmente tanto desde departamentos de Ingeniería de Telecomunicaciones como desde departamentos de Ingeniería Informática, de manera que los conceptos se suelen dividir según la tradición de estos departamentos. Como resultado, la mayor parte del estudiantado de telecomunicaciones no suele enfrentarse al aprendizaje de arquitecturas software, y la mayor parte del estudiantado de informática no aprende procesado digital de la señal o sistemas radio. El profesorado implicado se aproxima al dispositivo SDR incorporando la visión del departamento, considerando entonces que se trata, o bien de un sistema radio reconfigurable, o de un dispositivo que permite desarrollar software específico, cuando realmente los dispositivos SDR engloban todas las vertientes mencionadas (Rice y Col. 2019). El objetivo general es, tal y como se ha planteado en la introducción, acercar la docencia hacia una experimentación más realista de los sistemas de comunicaciones.

Los objetivos específicos del trabajo presentado son:

- Mejorar la motivación e implicación del alumnado. Este objetivo se centra en medir la mejora en la implicación del alumnado, de forma que perciban una mayor relevancia de las actividades que realizan en el laboratorio.

- Mejorar la energía de los estudiantes de GIT-UV. Este objetivo afecta a la asistencia y a la actitud de los estudiantes en las sesiones de laboratorio. El concepto energía está aquí relacionado con la capacidad de resiliencia del estudiantado frente a la resolución de problemas.

- Desarrollar un proyecto transversal a distintos cursos de la carrera y materias (Señales y Sistemas, Comunicaciones Digitales). De esta manera se cumplen con las indicaciones recogidas en sellos de calidad como EUR-ACE.

\section{Desarrollo de la innovación}

En el curso académico 2018-2019, se propone a un grupo de ocho estudiantes (cuatro parejas de trabajo en laboratorio), la participación de forma voluntaria en un proyecto de innovación docente donde, en lugar de realizar las prácticas estándar basadas en simulación, iban a desarrollar variaciones basadas en interfaces para trabajar con SDR. Uno de los grupos realizó una práctica con el programa GNURadio, siguiendo el modelo presentado en la Fig. 1. Concretamente, se trataba de una práctica para probar distintos parámetros relacionados con los códigos de repetición de un sistema con codificación de canal. La práctica se planteó inicialmente con una modulación de tipo Binary Phase-Shift Keying (BPSK) y los estudiantes se encargaron de ampliarla para que diera cabida también a la modulación Quadrature Amplitude Modulation (QAM) de $M=4$ posibles símbolos.

En el curso académico 2019-2020 la asignatura tiene 39 estudiantes matriculados, que se reparten en 3 grupos de laboratorio: AL1 (13 estudiantes), AL2 (16 estudiantes) y AL3 (10 estudiantes). El número final de estudiantes que realiza presencialmente los laboratorios es: AL1 (9 estudiantes), AL2 (14 estudiantes) y AL3 (6 estudiantes). Con estos datos, se decide que el grupo AL2 sea el grupo experimental que realizará la práctica basada en SDR, mientras que los grupos AL1 y AL3 realizarán la práctica simulada estándar, constituyendo los grupos de control y siendo el número de 


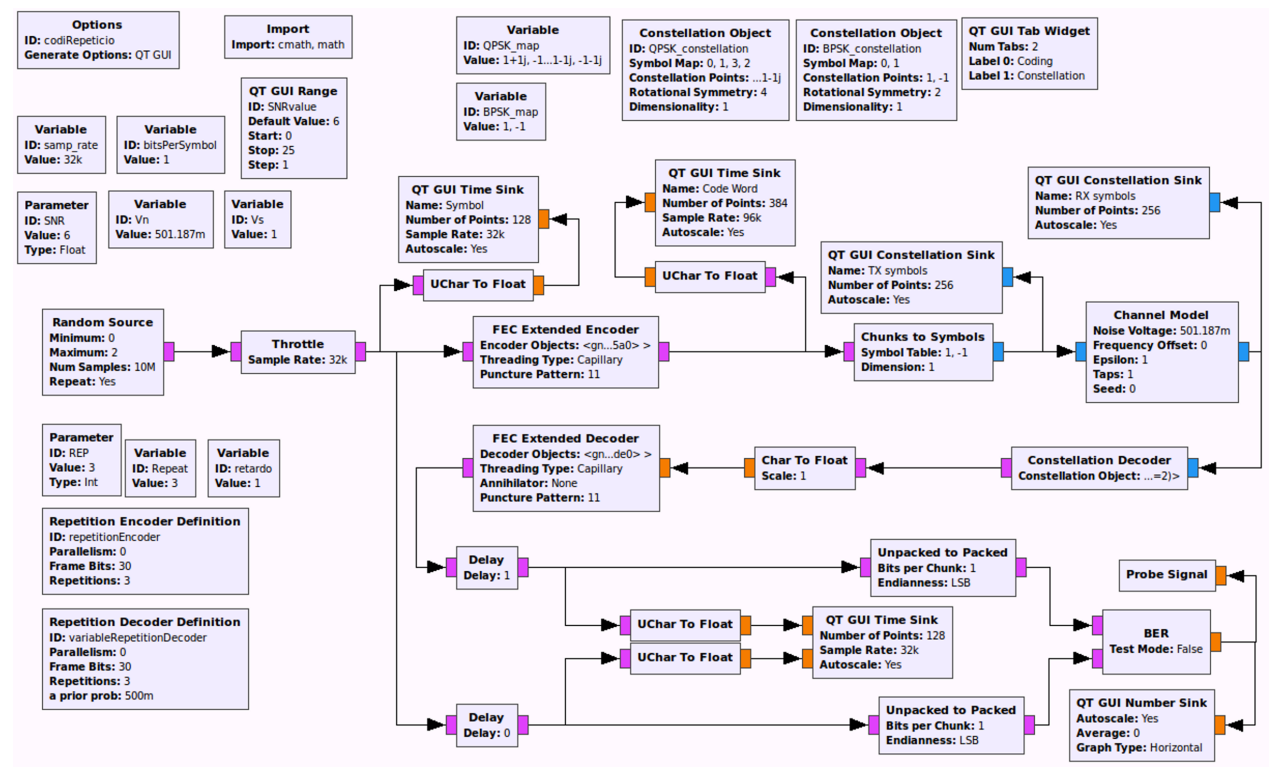

Fig. 1: Esquemático a implementar con GNURadio.

estudiantes agregado similar en ambos casos. Además, los grupos AL2 y AL3 realizan las prácticas en paralelo el mismo día de la semana y a la misma hora, mientras que el grupo AL1 las realiza otro día pero en la misma franja horaria. De esta forma, se intentan evitar aspectos que puedan influir de forma negativa en la evaluación de la innovación docente, como es el tener grupos muy numerosos en los laboratorios, o la realización de las prácticas en franjas horarias distintas. Puesto que los componentes de cada grupo no se seleccionan al azar, sino que se asignan a los grupos preexistentes, el diseño de esta innovación sería de tipo cuasi-experimental.

El grupo AL2 realiza la práctica planteada por el grupo voluntario en el curso académico 20182019, mientras que los grupos AL1 y AL3 realizan una práctica equivalente mediante simulación en el programa MATLAB, donde se programan todos los bloques necesarios en un sistema que implementa codificación de canal mediante códigos de repetición. Cabe destacar que la programación de todos los bloques del sistema es mucho más costosa en tiempo y menos intuitiva que la construcción del esquemático de la Fig. 1, donde básicamente hay que buscar, añadir, configurar y unir los distintos bloques del sistema. De esta forma, el estudiante utiliza menos tiempo del disponible en la sesión en aspectos puramente de programación, contando con más tiempo para analizar el impacto de los distintos parámetros de la comunicación en el comportamiento del sistema. Nótese que la sesión de laboratorio es de 2.5 horas. 


\section{Resultados}

Para evaluar el impacto del uso de los dispositivos SDR se ha adaptado un cuestionario que evalúa el compromiso en el trabajo (Schaufeli y Bakker 2004; García-Ros y Col. 2018). El motivo de seleccionar este cuestionario fue la necesidad de valorar de forma objetiva la implicación del alumnado, ya que en trabajos anteriores se utilizaron como variables para la evaluación las notas obtenidas en las prácticas de laboratorio, o los resultados de las encuestas realizadas por la unidad de calidad (Segura 2016; Soriano-Asensi 2019). El estudiantado valoró 10 preguntas utilizando una escala Likert entre 1 (nunca/casi nunca) y 5 (casi siempre/siempre):

- Q1 - En el laboratorio me siento lleno/llena de energía;

- Q2 - Pienso que las prácticas son relevantes y significativas;

- Q3 - El tiempo me parece que "vuela" mientras realizo las prácticas;

- Q4 - Me siento con gran fuerza y vigor durante las prácticas;

- Q5 - Me entusiasman las prácticas que se nos proponen;

- Q6 - Mientras realizo las prácticas me olvido de todas las cosas que pasan a mi alrededor;

- Q7 - El trabajo en el laboratorio me resulta ilusionante;

- Q8 - Tengo ganas de ir a clase cuando llego a la Universidad;

- Q9 - Me satisface trabajar con intensidad en el laboratorio;

- Q10 - En general, me siento muy satisfecho/a con las actividades que se nos proponen en el laboratorio.

La pregunta Q10 está relacionada con la satisfacción general del estudiantado. El resto de preguntas tienen como objetivo evaluar tres aspectos indicativos de la implicación académica: energía, relacionada con la capacidad de resiliencia del estudiantado frente a la resolución de problemas (Q1, Q4, Q8); absorción, relacionada con la capacidad del estudiantado para concentrarse en aquellas tareas que está realizando (Q3, Q6, Q9); y dedicación, relacionada con la percepción del estudiantado sobre la relevancia de las actividades que realiza (Q2, Q5, Q7). El cuestionario se realizó de forma previa a la interacción con los dispositivos SDR, y tras la realización de la práctica de laboratorio. Notese que los estudiantes son de tercer curso, por lo que ya han pasado previamente por diversas asignaturas con una metodología idéntica en las prácticas de laboratorio (simulación mediante el programa MATLAB) y, por tanto, pueden contestar de forma adecuada a algunas de las preguntas propuestas.

La Fig. 2 refleja las puntuaciones promedio, obtenidas en las respuestas de cada pregunta, en el muestreo previo y el muestreo posterior. Tal y como muestra la figura, la diferencia obtenida en los resultados agregados de los grupos de control AL1 y AL3 es positiva en todas las preguntas, a excepción de la pregunta Q4. La diferencia más apreciable se consigue en la pregunta Q6 en este caso. Por otro lado, en el caso del grupo experimental AL2, la diferencia obtenida es positiva en todas las preguntas a excepción de las preguntas Q3 y Q6, siendo la diferencia positiva más importante la conseguida en la pregunta Q7. En el caso de la pregunta Q3, el descenso es poco apreciable. El resultado obtenido en la pregunta Q10 (satisfacción general) es similar tanto en el grupo experimental como en los grupos de control.

La Fig. 3 muestra los resultados obtenidos cuando se agregan las respuestas a las preguntas según su categoría (energía, absorción y dedicación). En este caso, se puede observar cómo la realización de la práctica basada en SDR ha conseguido aumentar positivamente la diferencia (por encima de los resultados obtenidos en los grupos estándar) en los aspectos de energía y dedicación, mientras que 
C. Botella, A. Soriano-Asensi, J. Segura-Garcia, J. Perez, S. Felici-Castell, E. Navarro-Camba, M. García-Pineda y M. Montagud

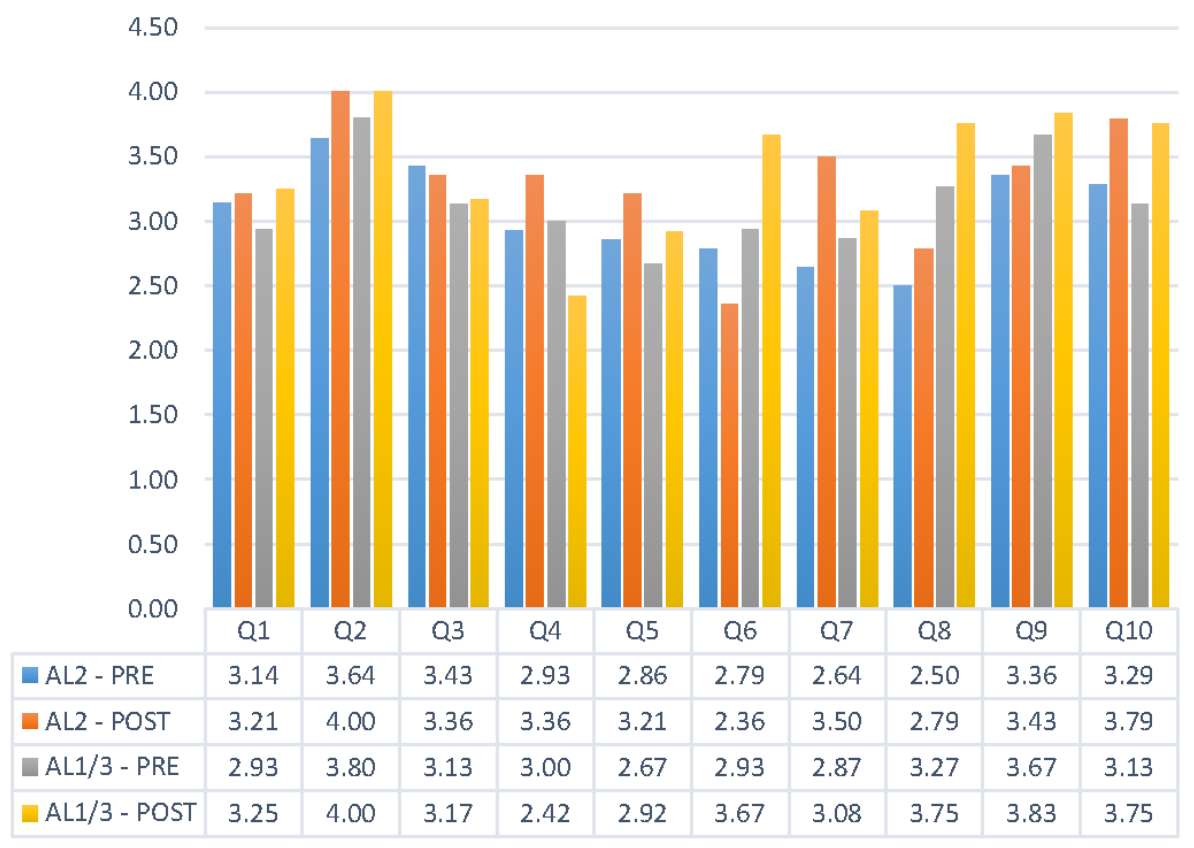

Fig. 2: Puntuaciones promedio para cada pregunta, antes y después de la realización de la sesión de laboratorio. Los resultados se muestran para el grupo experimental (AL2) y para la agregación de los grupos de control AL1 y AL3.

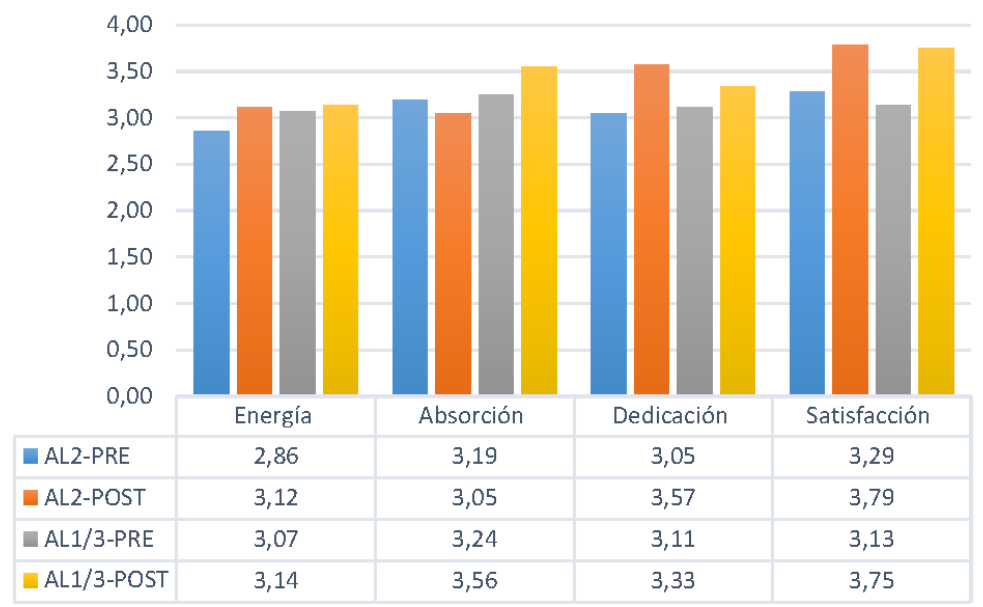

Fig. 3: Puntuaciones promedio agregadas para los aspectos de energía, absorción, dedicación y satisfacción general, antes y después de la realización de la sesión de laboratorio. Los resultados se muestran para el grupo experimental (AL2) y para la agregación de los grupos de control AL1 y AL3. 
ha disminuido ligeramente el aspecto de absorción. Cabe destacar que el descenso en la absorción viene dominado en el grupo experimental por la pregunta Q6. Una posible justificación a este resultado es la percepción por parte del estudiantado de la dependencia de un dispositivo hardware, de manera que hay más probabilidades de que surjan imprevistos que dificulten la realización de la práctica de forma satisfactoria (por ejemplo, mala recepción de la señal por un mal funcionamiento de la antena receptora, necesidad de configurar adecuadamente el programa con los paquetes de soporte para el hardware utilizado, etc).

Desde el punto de vista del profesorado, este tipo de iniciativas conlleva un alto coste temporal de preparación de los laboratorios, así como la necesidad de preparar trabajos alternativos para los estudiantes que no pueden asistir a la clase de forma presencial. Además, al utilizar dispositivos de tipo hardware, las prácticas deben acabarse de forma obligatoria durante la sesión de laboratorio, no hay posibilidad de finalizarlas fuera del laboratorio como así ocurre con las sesiones estándar simuladas.

Como trabajo futuro para el próximo curso académico, se contempla la posibilidad de evaluar si existe una correlación entre los resultados obtenidos en el cuestionario y otros ítems como las notas de las prácticas de laboratorio, e incluir herramientas que permitan recoger la opinión del profesorado implicado en el proceso.

\section{Conclusiones}

Este trabajo ha presentado el proceso de innovación llevado a cabo en los cursos académicos 20182019 y 2019-2020 en la asignatura Teoría de la Comunicación del Grado en Ingeniería Telemática de la Universitat de València. Esta línea se incluye dentro de un proyecto de innovación docente más amplio desarrollado en los últimos cinco años con el objetivo de actualizar las sesiones de laboratorio y acercarlas a prácticas más realistas a través de la introducción de dispositivos de radio definida por software. Específicamente, en el curso académico 2019-2020 se ha adaptado una práctica de laboratorio que modificó y amplió un grupo voluntario de estudiantes en el curso académico anterior. Para evaluar el impacto de forma sistemática y como paso previo a extender esta sesión a todos los grupos de laboratorio de la asignatura, se ha identificado un grupo experimental de laboratorio para realizar la práctica, trabajando el resto de grupos en una práctica alternativa equivalente. La evaluación del impacto indica que la experiencia ha sido positiva en las dimensiones de energía, dedicación y satisfacción general, disminuyendo ligeramente la capacidad de absorción del estudiantado. Desde el punto de vista del profesorado, estas herramientas requieren una mayor inversión en tiempo en la preparación de las sesiones de laboratorio. Por este motivo, es fundamental establecer un sistema de evaluación adecuado de la innovación para asegurar que la experiencia es positiva para el aprendizaje del estudiantado.

\section{Referencias bibliográficas}

Bilén, S.G. y Col. (mayo de 2014). "Software-defined radio: a new paradigm for integrated curriculum delivery". En: IEEE Communications Magazine 52.5, págs. 184-193.

García-Ros, R. y Col. (sep. de 2018). "The schoolwork engagement inventory: factorial structure, measurement invariance by gender and educational level, and convergent validity in secondary education (12-18) years." En: Journal of Psychoeducational Assessment 36.6, págs. 588-603. 
C. Botella, A. Soriano-Asensi, J. Segura-Garcia, J. Perez, S. Felici-Castell, E. Navarro-Camba, M. García-Pineda y M. Montagud

Gil Jiménez, V.P. y Col. (mayo de 2017). "Learning mobile communications standards through flexible software defined radio base stations". En: IEEE Communications Magazine 55.5, págs. 116-123.

Mitola, J. (mayo de 1992). "Software radios-survey, critical evaluation and future directions". En: Proc. IEEE National Telesystems Conference, (NTC'92). Washington DC, USA, págs. 15-23.

Rice, M. y Col. (nov. de 2019). "Teaching communications with SDRs: making it real for students". En: IEEE Communications Magazine 57.11, págs. 14-19.

Schaufeli, W. y A. Bakker (dic. de 2004). UWES-Utrecht Work Engagement Scale: Test manual. Department of Psychology, Utrecht University, Utrecht, The Netherlands.

Segura, J. y Col. (jul. de 2016). "Innovación en docencia de sistemas de comunicación en el Grado de Ingeniería Telemática de la UVEG". En: IN-RED 2016. II Congreso de Innovación Educativa y Docencia en Red. Valencia (Spain).

Soriano-Asensi, A. y Col. (jul. de 2019). "Aprendizaje basado en proyectos en los laboratorios de comunicaciones digitales". En: IN-RED 2019. V Congreso de Innovación Educativa y Docencia en Red. Valencia (Spain).

Stewart, R.W. y Col. (sep. de 2015). "A low-cost desktop software defined radio design environment using MATLAB, simulink, and the RTL-SDR". En: IEEE Communications Magazine 53.9, págs. 64-71.

Wyglinski, A.M. y Col. (ene. de 2016). "Revolutionizing software defined radio: case studies in hardware, software, and education". En: IEEE Communications Magazine 54.1, págs. 68-75. 